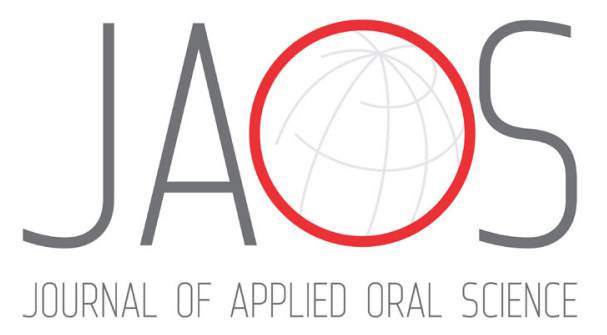

\title{
Effect of two corticotomy protocols on periodontal tissue and orthodontic movement
}

\section{Abstract}

Marcelo Lelis ZUPPARDO ${ }^{1}$

Milton SANTAMARIA JR ${ }^{2}$

Camila Lopes FERREIRA ${ }^{1}$

Mariéllen $\mathrm{LONGO}^{3}$

Joni Augusto CIRELLI ${ }^{4}$

Mauro Pedrine SANTAMARIA ${ }^{5}$

Maria Aparecida Neves JARDINI ${ }^{5}$
Submitted: January 13, 2020 Modification: April 23, 2020

Accepted: May 19, 2020

Corresponding address: Maria Aparecida Neves Jardin Eng. Francisco José Longo Ave, 777 - 12245-000 - São José dos Campos - SP - Brasil. phone: (55) 12-39479046 e-mail: jardini@ict.unesp.br
Objective: To compare two corticotomy surgical protocols in rats to verify whether they alter conventional orthodontic movement. Methodology: Sixty Wistar rats were divided into three groups - orthodontic movement (CG), orthodontic movement and corticotomy (G1) and orthodontic movement with corticotomy and decortication (G2) - and euthanized after 7 and 14 days. Tooth movement $(\mathrm{mm})$, bone volume fraction and bone volume ratio to total volume (BV/TV), and bone mineral density (BMD) were evaluated by micro-CT. The total amount of bone was measured in square millimeters and expressed as the percentage of bone area in the histomorphometry. The number of positive TRAP cells and RANK/RANKL/OPG interaction were also investigated. Results: Day 14 showed a statistically significant difference in orthodontic tooth movement in CG compared with $\mathrm{G} 1$ (7.52 mm; $\mathrm{p}=0.009)$ and $\mathrm{G} 2$ (7.36 mm; $\mathrm{p}=0.016)$. A micro-CT analysis revealed a difference between $C G, G 1$ and $G 2$ regarding $B V / T V$, with $G 1$ and $G 2$ presenting a lower BV/TV ratio at 14 days ( 0.77 and 0.73 respectively); we found no statistically significant differences regarding BMD. There was a difference in the total amount of bone in the CG group between 7 and 14 days. At 14 days, CG presented a significantly higher bone percentage than G1 and G2. Regarding TRAP, G2 had more positive cells at 7 and 14 days compared with CG and G1. Conclusion: Corticotomy accelerates orthodontic movement. Decortication does not improve corticotomy efficiency.

Keywords: Corticotomy. Orthodontic movement. Animal study.

1 Universidade Estadual Paulista (UNESP), Pós-graduação em Biopatologia Bucal, Curso de Odontologia - ICT, Disciplina de Periodontia, São José dos Campos, São Paulo, Brasil. ¿UNIARARAS, Centro Universitário Hermínio Ometto, Programa de Pós-Graduação em Ortodontia e Programa de Pós-Graduação em Ciências Biomédicas, Araras, Brasil.

${ }^{3}$ Universidade Estadual Paulista (UNESP), Curso de Odontologia - ICT, Pos-Doutorado, Disciplina de Periodontia, São José dos Campos, São Paulo, Brasil.

${ }^{4}$ Universidade Estadual Paulista (UNESP), Faculdade de Odontologia - FOAR, Disciplina de Periodontia, Araraquara, São Paulo, Brasil.

${ }^{5}$ Universidade Estadual Paulista (UNESP), Curso de Odontologia - ICT, Disciplina de Periodontia, São José dos Campos, São Paulo, Brasil. 


\section{Introduction}

With an increasing number of adult patients seeking orthodontic treatment, orthodontists are constantly searching for treatments to accelerate orthodontic movement that are predictable and have few complications. ${ }^{1}$ Such techniques include reducing the treatment period using self-ligating bracket systems; ${ }^{2}$ wires with memory (NiTi); ${ }^{3}$ direct electric currents or magnet; ${ }^{4}$ micro implants; ${ }^{5}$ surgical interventions ${ }^{6}$ and administration of local or systemic medications. $^{7}$

Among surgical interventions is corticotomy - an intentional bone injury limited to a cortical portion of the alveolar bone with minimal penetration into the medullary bone..$^{5}$ It is indicated to speed up corrective orthodontic treatment and facilitate executing mechanically difficult orthodontic movement, as well as to correct moderate to severe skeletal occlusions and decrease treatment time. 6,8,9

Fast orthodontic treatments are essential as shorter treatments are more acceptable to patients, and long-term treatments have been associated with negative results, such as an increased risk of dental cavities, ${ }^{10}$ periodontal disease,${ }^{11}$ root resorption ${ }^{12}$ and pulp reactions. ${ }^{13}$

Accelerated osteogenesis has sparked interest in the orthodontic community and animal studies have demonstrated the biological reactions of bone remodeling and periodontal tissues when associated with corticotomy and orthodontic movement. ${ }^{1}$ During bone remodeling, a phenomenon known as regional acceleratory phenomenon (RAP) occurs. Frost ${ }^{14}$ (1983) described it as faster tooth movement due to reduced resistance of the cortical bone through the surgical procedure; Yaffe, Fine, Binderman ${ }^{15}$ (1994) described RAP as a temporary explosion of localized remodeling of soft and hard tissues, i.e. a regeneration that rebuilds the bone, thus restoring its normal state. Sebaoun, et al. ${ }^{16}$ (2008) observed that RAP increases bone metabolism, activates osteoclasts and osteoblasts, and decreases bone density.

Baloul, et al. ${ }^{17}$ (2011) compared orthodontic movement with and without alveolar corticotomy, using tomography and molecular methods, and found that the corticotomy group achieved faster initial tooth displacement. Dibart, et al. ${ }^{18}$ (2014) used a minimally invasive technique (piezo-incision) that resulted in accelerated orthodontic movement and less extensive and traumatic surgical treatment.

The use of corticotomy in orthodontic treatment is increasing and studies have mainly investigated clinical cases with many variations in both surgical and orthodontic protocols. ${ }^{9}$ Corticotomy procedures can produce statistically and clinically meaningful temporary increases in the rate of orthodontic tooth movement with minimal side-effects. ${ }^{19}$

Thus, this study aimed to evaluate two surgical corticotomy protocols by an experimental model involving rats. The null hypothesis was that there is a similarity in orthodontic movement between two treatments with different amounts of surgical injury.

\section{Methodology}

The study was approved by the CEUA $n^{0} 08 / 2015-$ ICT-SJC-UNESP. Sixty male Wistar rats (Rattus norvegicus, albinos) aged 90 days and weighing 300 $\mathrm{g}$ on average were kept in plastic cages, at room temperature $\left(22^{\circ} \mathrm{C}\right)$ for a 12 -hour light cycle with standard diet and water ad libitum.

They were randomly divided into three groups: CG $(n=20)$ received conventional orthodontic treatment; G1 $(n=20)$ received a less invasive corticotomy treatment followed by orthodontic treatment; and G2 $(n=20)$ received corticotomy with decortication and orthodontics, a more invasive protocol.

\section{Sample size}

The sample size needed to determine tooth movement (primary outcome) calculated by a previous study ${ }^{20}$ with an alpha level of $5 \%$ and power of $80 \%$ was 17 animals/group. To ensure sufficient samples, the final $n$-sample comprised 20 animals/group.

\section{Orthodontic appliance placement}

The orthodontic devices were installed on the animals' lower first molar, as previously described. ${ }^{21}$ After anesthesia, a $0.12 \mathrm{~mm}$ alloy wire (Morelli, Sorocaba, SP, Brazil) was inserted between the first and second right molars. A closed-coil steel spring of $3 \mathrm{~mm}$ in length (Morelli, Sorocaba, SP, Brazil) was pre-calibrated so as to release a continuous force, and attached to the lower incisors with a light-curing resin (3M ESPE, USA), thus causing a spring pull of 40-g $\mathrm{g}^{22}$ of force. A 40-g force was applied on the first molar (point of force application) and lower incisors 
(anchor point) to allow moving the first molar to the mesial direction

\section{Surgical procedure}

G1 and G2 animals underwent surgery just after installation of the orthodontic appliance. G1 received trichotomy of the masseter muscle region, and the area was disinfected with chlorhexidine digluconate. A $10-\mathrm{mm}$ incision from the labial commissure region towards the mandible angle was performed, exposing the buccal cortical bone of the first lower molar roots. ${ }^{23}$ Corticotomy was performed using the tip (W1-0-CVDentus) of a piezo-surgical device (DentSurg CVDentus, Brazil) under saline irrigation, creating two vertical grooves and a horizontal one, with a U-shaped design in the apical region of the first lower molar (Figure1a).

G2 received corticotomy with a $\mathrm{U}$-shape in the apical region of the first lower molar, with part of the cortical bone (decortication) being removed from the center of the groove (Figure $1 b$ ) with a spherical tip (R4). The muscle was repositioned and sutured with vicryl 4-0 and the skin was sutured with 3-0 silk. ${ }^{21}$

The animals were euthanized through transcardial perfusion at 7 and 14 days. ${ }^{24}$ Their hemi-mandibles were removed and stored in a buffered $4 \%$ paraformaldehyde solution for 72 hours.

All analyses were evaluated by a blinded and previously calibrated examiner.

\section{Macroscopic analysis of orthodontic movement $(\mathrm{mm})$}

We measured the amount of orthodontic movement at 7 and 14 days. Tooth movement from the mesial first molar to the distal third molar was quantified using a digital caliper (Mitutoyo Absolute Digimatic Model CD-6 "CX-B, Brazil) after dissecting the jaws.

\section{Computerized microtomography (micro-CT) analysis}

CG, G1, and G2 hemi mandibles were scanned using a high-resolution microtomography (Sky Scan1176 Bruker MicroCT Actselaar Belgium). Sections of $9 \mu \mathrm{m}$ thickness (50 ky, $500 \mu \mathrm{m}$ ) were made and reconstructed using the NRecon software (SKYcan, 2011,1.6.6.0). Misalignment compensation values were calculated individually.

The CTAnalyzer software delimited the region of interest using a grayscale (25-90 tons) which was used to evaluate changes in alveolar bone volume in the bifurcation root areas of the lower first molar in 3D slices. The parameters were determined according to Nogueira, et al. ${ }^{25}$ (2017): TV: total tissue volume measured by contours in the region of interest; $\mathrm{BV}$ : volume of mineralized tissue; BV/TV: bone volume fraction, ratio of bone volume to total volume; BMD: bone mineral density.

\section{Histomorphometric analysis}

The samples were demineralized in a $10 \%$ EDTA solution at $7.8 \mathrm{pH}$ and immersed in paraffin, then cut into transverse plane allowing visualization of the roots and the periodontal ligament of the first mandibular tooth. Five semi-sequential slices $(5 \mu \mathrm{m})$ of the furcation region spaced $125 \mu \mathrm{m}$ apart were stained with hematoxylin and eosin. Images were captured at 25x magnification using an Axioplan 2 microscope and Axiovision Rel 4.7 image capture software. Images were renamed to appropriately blind the examiner. Histomorphometric analysis was performed according to Dibart, et al. ${ }^{18}$ (2014): a geometric area was delimited using the four center roots creating the interradicular area in which the bone tissue was measured in $\mathrm{mm}^{2}$ and the outcomes were expressed as percentages (Figure 2).
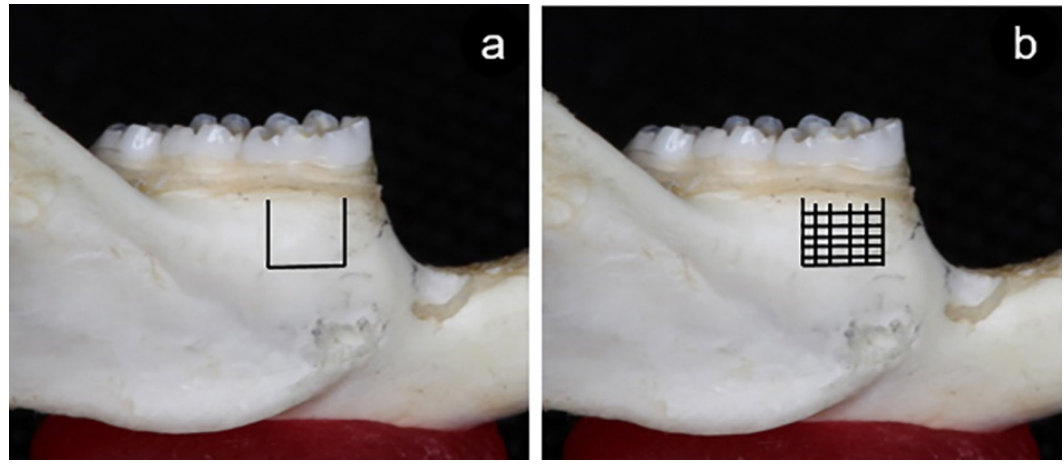

Figure 1- Schematic drawing of corticotomy(a) and decortication (b) 


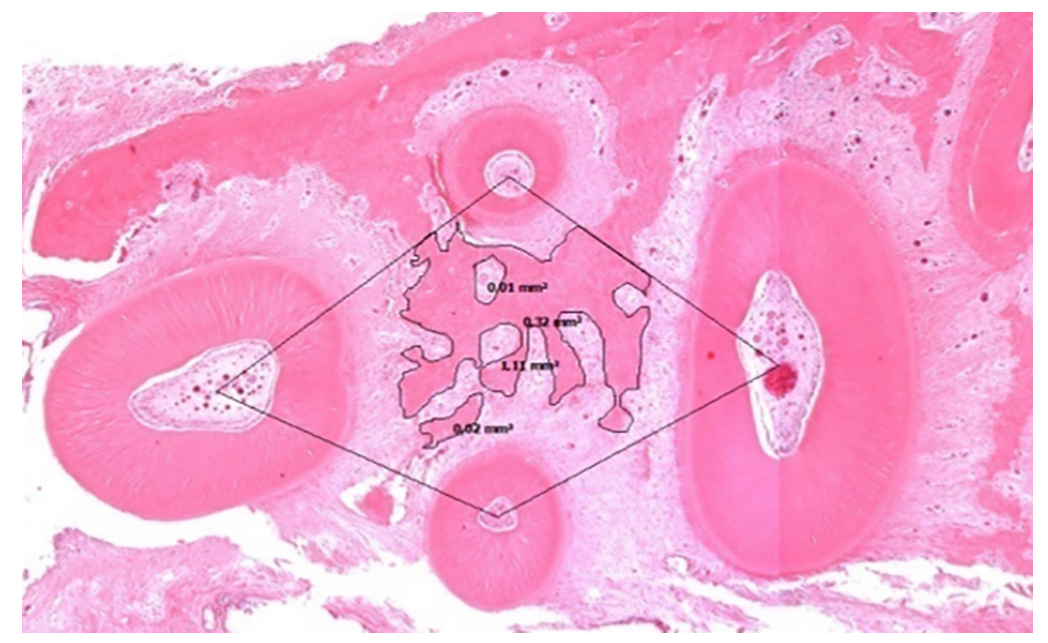

Cross-section of the first molar mandibular with geometric area and total amount of bone outlined.

Figure 2- Delimitation of histomorphometric analyses

\section{Immunohistochemistry analyses (RANK, RANKL, OPG, and TRAP)}

Before the immunohistochemical analyses, paraffin blocks were cut into serial cross-sections with $4 \mu \mathrm{m}$ thickness and placed on slides previously treated with 3-aminopropyltriethoxysilane (Sigma Chemical, USA).

The samples ( $n=6 /$ group/antibody) were immunohistochemically processed as previously described. ${ }^{21}$ We performed indirect immunoperoxidase using the following primary antibodies: anti-receptor activator of nuclear factor-KB (anti-RANK, 1:100, ab13918, abcam, Cambridge, MA, USA), anti-receptor activator of nuclear factor kappa-B ligand (antiRANKL,1:100, ab45039, abcam), anti-osteoprotegerin (anti-OPG, 1:400; ab73400, abcam) and anti-tartrate resistant Acid Phosphatase (Anti-TRAP, 1:100; SC30833, Santa Cruz). For the negative control, one of the slices was incubated only with the antibody diluent (Spring-codADS-125). Positive control concerned the development of a golden-brown precipitate as the final product of the bone tissue reaction, specifically indicating osteoblasts.

A semi-quantitative immunolabeling analysis was performed for RANK, RANKL, and OPG antibodies. The region of interest was the mesial area of the vestibular root of the lower right first molar, i.e. the pressure region and adjacent to the corticotomy and zone of decortication. The established score criteria for these antibodies were applied according to previous studies $^{21}$ : score 0 - absence of immunolabeling; score 1 - low immunolabeling; score 2 - moderate immunolabeling; score 3 - high immunolabeling.

We defined TRAP immunolabeling as presence of brownish color in the cytosolic compartment of positive cells, which was performed by counting TRAP-positive cells located in the mesial area of the vestibular root (pressure region) and adjacent to the corticotomy and zone of decortication. The number of osteoclasts and pre-osteoclasts stained with TRAP was counted within the geometric center of the four roots of the first molar. ${ }^{21}$

All images were captured at 400x magnification using an Axioplan 2 microscope and Axiovision Rel 4.7 image capture software. The images were blinded and quantified using Image $\mathrm{J}$.

\section{Statistics}

Prior to each analysis, the examiner was calibrated using the Pearson test $(k=0.89)$ and blinded to all groups. The statistical analyses were performed using Sigma Plot 12.0. Descriptive statistics consisted of mean and standard deviation, with sample normality verified by the Shapiro-Wilk test. The Mann-Whitney test was used for intragroup comparison concerning the macroscopic measurements of movement; the Kruskal-Wallis test was used for intergroup analysis. The micro-CT, histomorphometry and immunohistochemistry results were analyzed by twoway ANOVA at $5 \%$ significance with Tukey test for post-hoc analysis.

\section{Results}

\section{Macroscopic measurement of orthodontic movement}

Groups G1 and G2 showed statistically significant differences in orthodontic movement compared with 
CG, which received the orthodontic appliance only $(p<0.01)$ (Table 1).

Table 1- Measurements of orthodontic movement in right mandibles measured in millimeters with a digital caliper

\begin{tabular}{cccc}
\hline Period & CG & G1 & G2 \\
& RM & RM & RM \\
\hline 7 Days & $6.98 \pm 0.19^{\mathrm{Aa}}$ & $7.12 \pm 0.25^{\mathrm{Aa}}$ & $7.18 \pm 0.16^{\mathrm{Aa}}$ \\
\hline 14 Days & $7.21 \pm 0.13^{\mathrm{Aa}}$ & $7.52 \pm 0.36^{\mathrm{Ab}}$ & $7.36 \pm 0.13^{\mathrm{Ab}}$ \\
\hline
\end{tabular}

Uppercase letters: statistically significant difference in the intragroup comparison, Mann-Whitney. Lower case letters: statistically significant difference in the intergroup comparison, Kruskal-Wallis. RM: right mandible.

\section{Computerized microtomography}

Regarding BV/TV, all groups showed no significant intragroup differences between 7 and 14 days; however, at 7 days, CG differed significantly from $\mathrm{G} 2$, and $\mathrm{G} 1$ was similar to $\mathrm{CG}$ and $\mathrm{G} 2$. At 14 days, CG differed significantly from $G 1$ and $G 2$. As for $B M D$, there were no significant inter- or intragroup differences (Table 2).

Figure 3 shows representative images of the first molar and region of interest obtained from the CG, G1, and G2 groups at 7 and 14 days.

Table 2- Micro-CT measurements of bone mineral fraction and bone mineral density

\begin{tabular}{|c|c|c|c|c|c|}
\hline & \multirow[b]{2}{*}{ Groups } & \multicolumn{2}{|c|}{7 days } & \multicolumn{2}{|c|}{14 days } \\
\hline & & $M \pm S D$ & $\mathrm{Me}$ & $\mathrm{M} \pm \mathrm{SD}$ & Me \\
\hline \multirow{3}{*}{$\mathrm{BV} / \mathrm{TV}$} & CG & $0.73 \pm 0.03^{\mathrm{Aa}}$ & 0,72 & $0.87 \pm 0.04^{\mathrm{Aa}}$ & 0,88 \\
\hline & $\mathrm{G} 1$ & $0.77 \pm 0.01^{\mathrm{Aab}}$ & 0,77 & $0.77 \pm 0.11 \mathrm{~A}^{\mathrm{Ab}}$ & 0,78 \\
\hline & G2 & $0.64 \pm 0.11^{\mathrm{Ab}}$ & 0,63 & $0.73 \pm 0.03^{\mathrm{Ab}}$ & 0,75 \\
\hline \multirow{3}{*}{ BMD } & CG & $0.82 \pm 0.04^{\mathrm{Aa}}$ & 0,81 & $0.95 \pm 0.07^{\mathrm{Aa}}$ & 0,97 \\
\hline & G1 & $0.90 \pm 0.01^{\mathrm{Aa}}$ & 0,9 & $0.92 \pm 0.10^{\mathrm{Aa}}$ & 0,94 \\
\hline & G2 & $0.80 \pm 0.11^{\mathrm{Aa}}$ & 0,81 & $0.87 \pm 0.04^{\mathrm{Aa}}$ & 0,88 \\
\hline
\end{tabular}

Uppercase letters: statistically significant difference in intragroup comparison by two-way ANOVA. Lower case letters: statistically significant difference in intergroup comparison by two-way ANOVA. M: mean; SD: standard deviation; Me: median; BV/TV: bone volume/ total volume; BMD: bone mineral density.

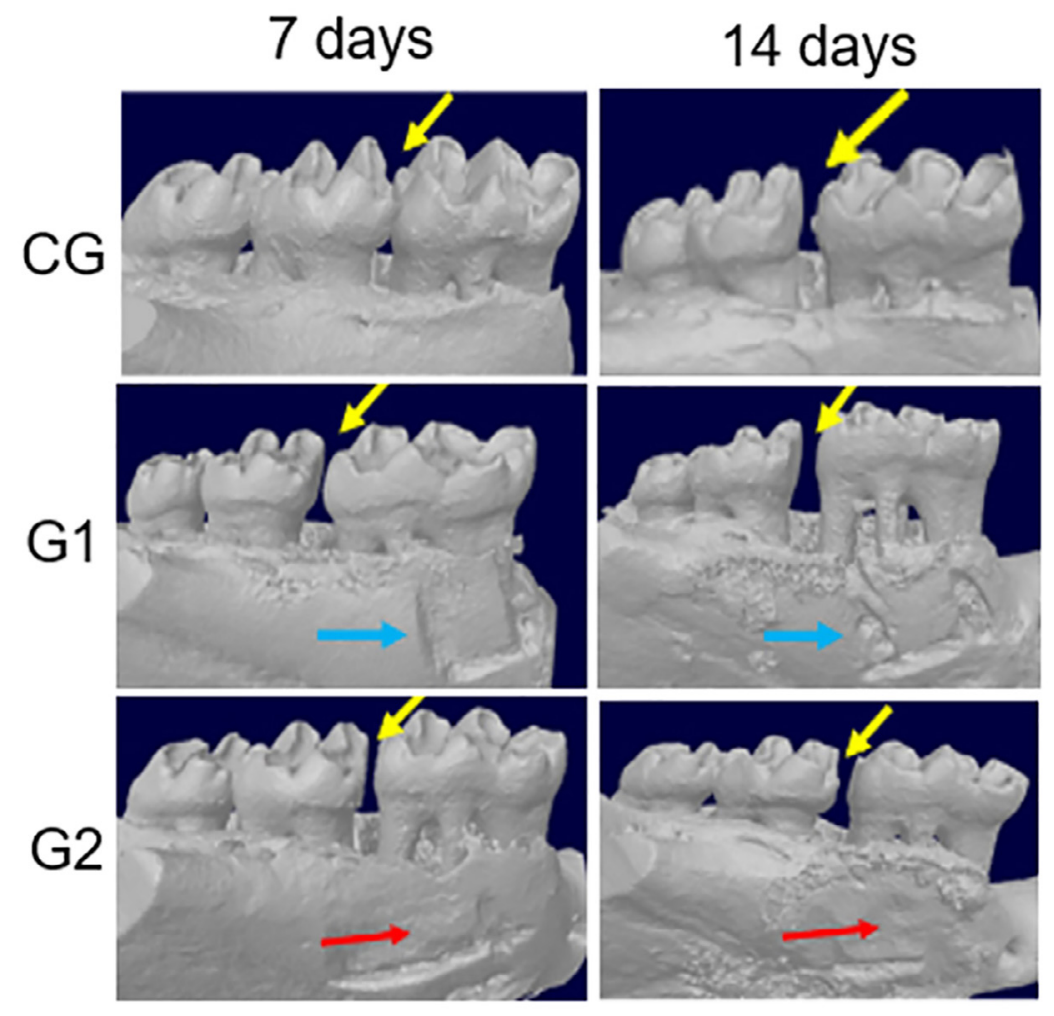

Images represent the right mandible teeth, where corticotomy (G-blue arrow), and corticotomy and decortication (G2-red arrow) were performed. Yellow arrows indicate the space created between the first and second molars in the vestibular region of the transverse plane.

Figure 3- Transverse plane images performed on high-resolution microcomputer tomography 


\section{Histomorphometric analysis}

The histomorphometric analysis revealed a significant difference in the total amount of bone present in CG at 14 days when compared with the $7^{\text {th }}$ day $(p=0.001)$. Other groups exhibited no such behavior. The intergroup analysis showed no significant differences at 7 days; however, at 14 days, CG presented a significantly higher total amount of bone than $G 1(p=0.003)$ and $G 2(p=0.001)$. Regarding the percentage of bone volume, the intragroup

Table 3- Histomorphometric measurements of the delineated geometric area in $\mathrm{mm}^{2}$

\begin{tabular}{|c|c|c|c|c|c|}
\hline & \multirow[b]{2}{*}{ Groups } & \multicolumn{2}{|c|}{7 days } & \multicolumn{2}{|c|}{14 days } \\
\hline & & $M \pm S D$ & Me & $M \pm S D$ & Me \\
\hline \multirow{3}{*}{ Total amount of bone $\left(\mathrm{mm}^{2}\right)$} & CG & $0.35 \pm 0.19^{A a}$ & 0,38 & $0.54 \pm 0.15^{\mathrm{Ba}}$ & 0,6 \\
\hline & G1 & $0.35 \pm 0.16^{\mathrm{Aa}}$ & 0,35 & $0.41 \pm 0.17^{A b}$ & 0,38 \\
\hline & $\mathrm{G} 2$ & $0.36 \pm 0.15^{\mathrm{Aa}}$ & 0,38 & $0.40 \pm 0.17^{A b}$ & 0,43 \\
\hline \multirow{3}{*}{$\%$ Bone } & CG & $38.3 \% \pm 17 \%$ Aa & $42 \%$ & $49 \% \pm 11 \%$ Аa & $52 \%$ \\
\hline & G1 & $33.9 \% \pm 13 \%$ Аa & $35 \%$ & $39 \% \pm 12 \%{ }^{A b}$ & $37 \%$ \\
\hline & $\mathrm{G} 2$ & $35.2 \% \pm 13 \% \mathrm{Aa}$ & $37 \%$ & $38 \% \pm 15 \%$ Ab & $43 \%$ \\
\hline
\end{tabular}

uppercase letters: statistically significant difference in intragroup comparison by two-way ANOVA. Lowercase letters: statistically significant difference in intergroup comparison by two-way ANOVA. M: mean; SD: standard deviation; Me: median.
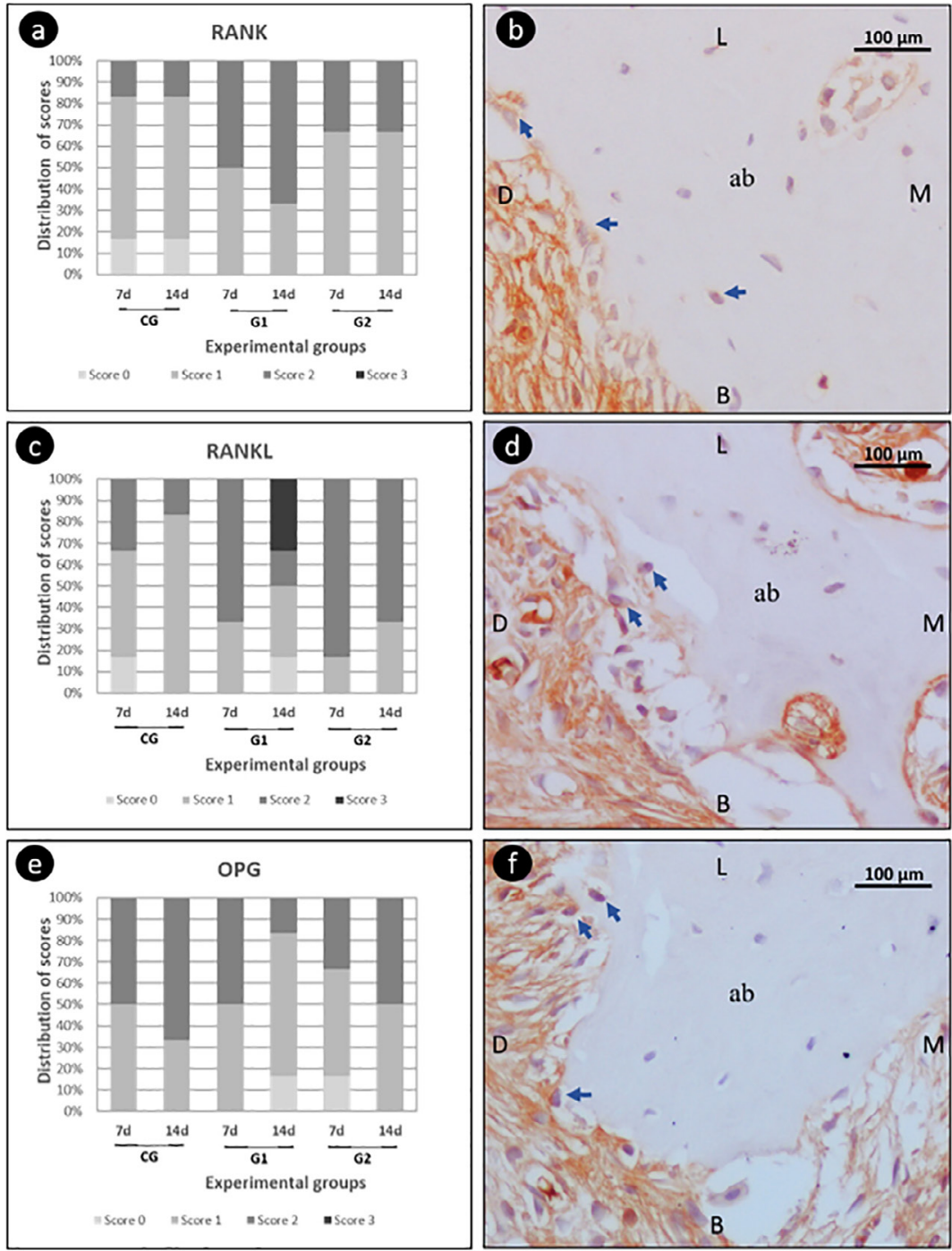

Immunolabeling score for RANK(a), RANKL(c), and OPG(e); photomicrographs evidencing the immunolabeling pattern for RANK(b); RANKL(d); and OPG(f). Blue arrows show immunolabeling cells. Abbreviations: ab: alveolar bone; L: lingual; B: buccal: M: mesial; D: distal 400x. Hematoxylin from Harris.

Figure 4- RANK, RANKL, OPG immunolabeling 


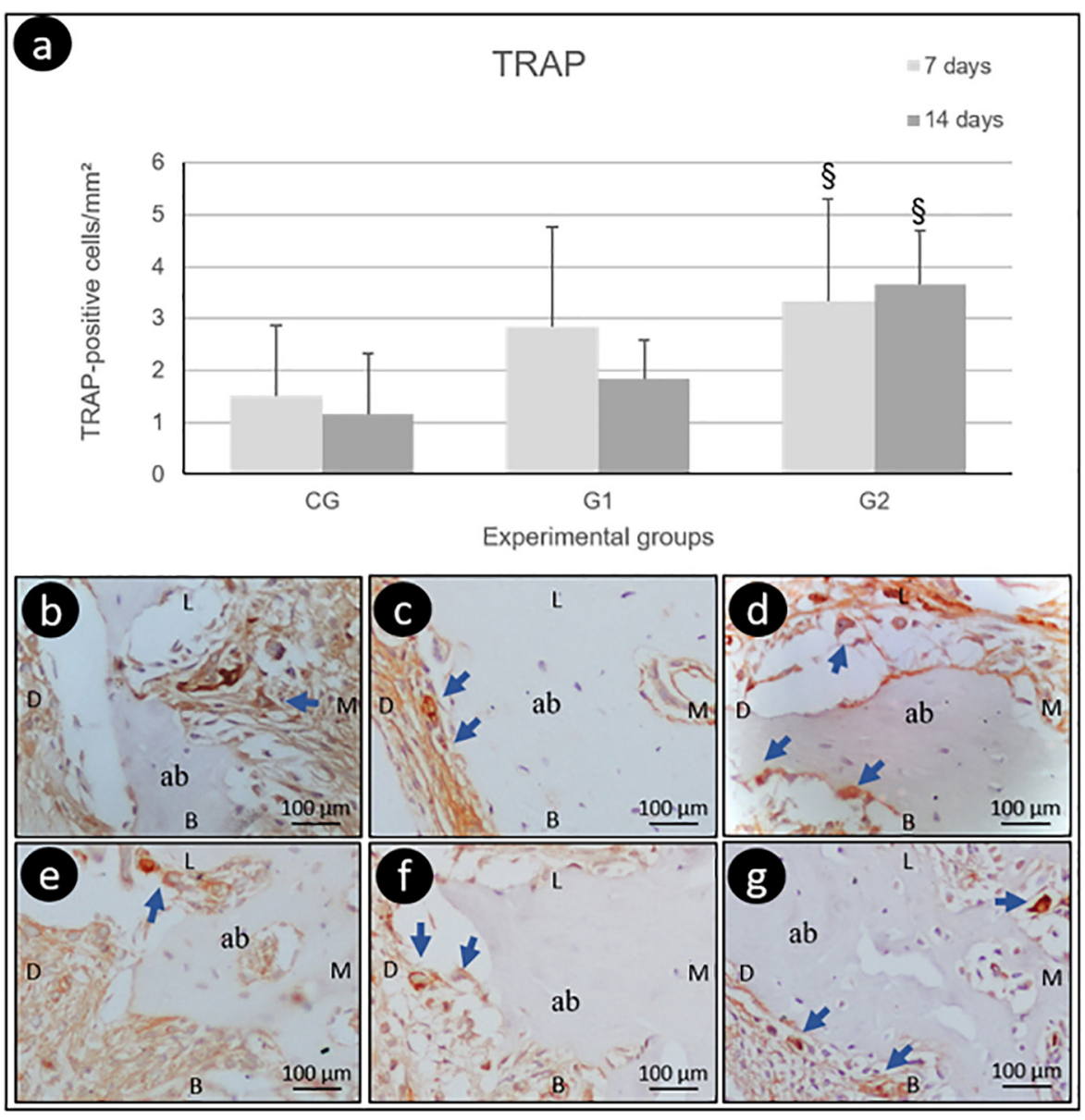

TRAP-positive cells $/ \mathrm{mm}^{2}$ in different experimental groups and periods (a); photomicrographs evidencing the immunolabeling pattern for TRAP (blue arrows) in the CG 7d (b), CG 14d (c), G1 7d (d), G1 14d (e), G2 7d (f), G2 14d (g). Abbreviations: ab, alveolar bone; L: lingual; B: buccal: M: mesial; D: distal; §, statistically significant difference of CG and G1 groups in the same period. 400x. Hematoxylin from Harris.

Figure 5- Mean and standard deviation of TRAP immunolabeling analysis showed no differences between times in any of the groups. In the intergroup analysis, CG had a significantly higher percentage of bone volume than $\mathrm{G} 1(\mathrm{p}=0.004)$ and $\mathrm{G} 2(\mathrm{p}=0.002)$ at 14 days (Table 3$)$.

\section{Immunohistochemical analyses (RANK, RANKL, OPG, and TRAP)}

In the immunohistochemical markers analysis, absolute values of RANK, RANKL, and OPG showed no significant differences in intra- and intergroup comparisons (Figure 4). In the TRAP analysis, G2 presented more positive cells, both at 7 days $(p=0.04)$ and 14 days $(p=0.04)$, compared with CG and G1 (Figure 5).

\section{Discussion}

Some studies suggest that corticotomy be performed as a way of accelerating orthodontic movement to reduce total treatment period. Therefore, it was hypothesized that combining orthodontic movement with corticotomy surgery could increase the amount of tooth movement. We compared two surgical protocols: one with corticotomy and another with corticotomy and decortication, in an experimental model to evaluate the process of bone remodeling at the tomographic, histological and immunohistochemical levels.

We chose rats as an experimental model because this is a relatively well-established model due to its low cost, easy storage, good availability and easy logistics. ${ }^{25}$ However, some limitations must be carefully evaluated when extrapolating the results of an experimental model in rats to humans. The use of a stainless-steel spring resulted from the application of high physiological forces during the process of bone remodeling, since the loss of the initial part of the applied force allows a favorable reaction in the periodontal tissues, closer to that desired in clinical practice, and has been widely used in many 
experimental studies. ${ }^{4,26,27,28}$ An initial force of $40 \mathrm{~N}$ is sufficient for tooth movement in this animal model and any loss of force intensity does not influence the analysis of tissue reactions in this experimental design.

The analysis of orthodontic movement in the different periods under study, whether associated with surgical procedures or not, revealed a statistically significant difference $(p<0.01)$, confirming the validity of the experimental model in the literature concerning induced tooth movement. ${ }^{27,28}$ Assessing two time points during experimental tooth movement is important as the literature describes a drastic reduction of osteoclasts at 7 days and 14 days in the duration of a remodeling cycle. ${ }^{29}$ An electronic caliper helped to measure the linear displacement being an commonly used method, ${ }^{21,27,30,31,32}$ and micro-CT analyses evaluated bone microarchitecture and BMD.

In this study, both groups that received corticotomy experienced accelerated orthodontic movement, and although one surgical procedure was more invasive than the other, both showed a similar orthodontic movement rate. This may relate to the use of the piezo-incision, with its micrometric and selective slices; the piezo-electric blade allows safe and precise osteotomies with no osteonecrosis damage. Two systematic reviews ${ }^{9,19}$ showed that corticotomy can increase the orthodontic tooth movement rate due to an altered physiological response through the RAP with minimal side effects. Complications include root resorption, loss of tooth vitality, periodontal sequelae, dentin hypersensitivity, pain and swelling. More studies evaluating the side effects are needed to reach a solid consensus on its use.

The micro-CT analysis revealed no significant difference in the BV/TV between 7 and 14 days within any of the groups; but when we performed an intergroup comparison, both at 7 and 14 days, CG presented a higher BV/TV than $\mathrm{G} 1$ and $\mathrm{G} 2$. A decrease in BV/TV can be attributed to transient osteopenia, which occurs after bone lesions. Regarding BMD, there was no significant difference between groups, but the changes that occurred may suggest an increase in spinal cord space, an influx of inflammatory cells, and increased vascularization in the region. ${ }^{17}$

The similar BMD indicates that the observed bone pattern remained harmonic. Although BDM remained unaltered, the surgical procedure was sufficient to accelerate orthodontic movement in $\mathrm{G} 1$ and $\mathrm{G} 2$. These results corroborate a study conducted by
Dibart, et al. ${ }^{18}$ (2014) who provided the first scientific evidence on the role of osteoclastic and osteoblastic activity associated with corticotomy and orthodontic movement.

The histomorphometric results were consistent with those shown by micro-CT images. We can understand the morphological processes by investigating biomarkers of bone remodeling and maturation.

Orthodontic forces promote cellular responses of hyalinization in the periodontal ligament, which induce bone resorption on the pressure side and bone deposition on the traction side. ${ }^{28}$ This process involves the induction of osteoclasts by the RANK-RANKL pathway and various inflammatory cytokines. RANKL/ OPG binding favorably modulates osteoclastogenesis and is considered an important factor in controlling bone resorption. 33

Based on the immunohistochemical analysis of RANK/RANKL/OPG biomarkers, our study showed greater expression of RANKL and its balance with OPG in animals that underwent corticotomy, despite the lack of biomarker differences, probably due to the time-length evaluated. Osteoblast activity was verified when the OPG was tested, and the maturation of osteoclasts with the analysis and quantification of TRAP in these cells. ${ }^{34}$ However, the animals showed greater maturation of osteoclasts and favored bone remodeling, as confirmed by the number of TRAPpositive cells, especially in G2.

This study suggests that although the most invasive technique (G2) resulted in the highest number of osteoclasts in the region of tooth movement, RANKL/ OPG expression indicates a balance in the modulation of bone differentiation in both experimentally corticotomized groups. Allowing one to infer that the less invasive technique promoted similar clinical results regarding its biological effect and the clinical displacement of the tooth. Thus, the adverse effects of periodontal ligament hyalinization and subsequent presence of dental resorption can be minimized, even when performing more conservative corticotomy procedures, increasing tooth movement efficiency.

\section{Conclusion}

According to our results, corticotomy accelerates orthodontic movement. Adding decortication to corticotomy does not improve its efficiency, suggesting 
that the less invasive technique should be selected for such purposes.

\section{Acknowledgements}

São Paulo Research Foundation FAPESP 2015/26523-5 and 2016/17141-4.

National Council for Scientific and Technological Development from Brazil, National Institute of Science and Technology Complex Fluids (INCT-FCx) - CNPq 465259/2014-6.

\section{Authors' contributions}

Zuppardo, Marcelo Lelis: Data curation (Equal); Methodology (Equal); Project administration (Equal); Writing-original draft (Equal). Santamaria Jr, Milton: Data curation (Equal); Formal analysis (Equal); Methodology (Equal); Visualization (Equal); Writingreview \& editing (Equal). Ferreira, Camila Lopes: Data curation (Equal); Methodology (Equal); Resources (Equal); Validation (Equal). Longo, Mariéllen: Data curation (Equal); Methodology (Equal); Resources (Equal); Writing-original draft (Equal). Cirelli, Joni Augusto: Data curation (Equal); Methodology (Equal); Visualization (Equal); Writing-review \& editing (Equal).

Santamaria, Mauro Pedrine: Formal analysis (Equal); Methodology (Equal); Visualization (Equal). Jardini, Maria Aparecida Neves: Conceptualization (Equal); Funding acquisition (Equal); Investigation (Equal); Methodology (Equal); Supervision (Equal); Writing-original draft (Equal); Writing-review \& editing (Equal).

\section{References}

1-Dab S, Chen K, Flores-Mir C. Short- and long-term potential effects of accelerated osteogenic orthodontic treatment: a systematic review and meta-analysis. Orthod Craniofac Res. 2019;22(2):61-8. doi: 10.1111/ocr.12272

2- Chen M, Li ZM, Liu X, Cai B, Wang DW, Feng ZC. Differences of treatment outcomes between self-ligating brackets with microimplant and headgear anchorages in adults with bimaxillary protrusion. Am J Orthod Dentofacial Orthop. 2015;147(4):465-71. doi: 10.1016/j. ajodo.2014.11.029

3- Gravina MA, Brunharo IH, Canavarro C, Elias CN, Quintão CC. Mechanical properties of NiTi and CuNiTi shape-memory wires used in orthodontic treatment. Part 1: stress-strain tests. Dental Press J Orthod. 201318(4):35-42. doi: 10.1590/S2176-94512013000400007 4- Spadari GS, Zaniboni E, Vedovello SA, Santamaria MP, Amaral ME, Santos GM, e tal. Electrical stimulation enhances tissue reorganization during orthodontic tooth movement in rats. Clin Oral Investig. 2017;21(1):111-20. doi: 10.1007/s00784-016-1759-6
5- Mostafa YA, Fayed MM, Mehanni S, ElBokle NN, Heider AM. Comparison of corticotomy-facilitated vs standard tooth-movement techniques in dogs with miniscrews as anchorage units. Am J Orthod Dentofacial Orthop. 2009;136(4):570-7. doi: 10.1016/j. ajodo.2007.10.052

6- Wilcko WM, Wilcko T, Bouquot JE, Ferguson DJ. Rapid orthodontics with alveolar reshaping: two case reports of decrowding. Int J Periodontics Restorative Dent. 2001;21(1):9-19.

7- Lee W. Experimental study of the effect of prostaglandin administration on tooth movement with particular emphasis on the relationship to the method of PGEI administration. Am J Orthod Dentofacial Orthop.1990;98(3):231-41. doi: 10.1016/s08895406(05)81600-2

8- Sukurica Y, Karaman A, Gürel HB, Dolanmaz D. Rapid canine distalization through segmental alveolar distraction osteogenesis. Angle Orthod. 2007;77(2):226-36. doi: 10.2319/0003-3219(2007)077[0226:RCD TSA]2.0.CO;2

9- Gil AP, Haas OL Jr, Méndez-Manjón I, Masiá-Gridilla J, VallsOntañón A, Hernández-Alfaro $F$, et al. Alveolar corticotomies for accelerated orthodontics: a systematic review. J Craniomaxillofac Surg. 2018;46(3):438-45. doi: 10.1016/j.jcms.2017.12.030

10- Richter $A E$, Arruda AO, Peters MC, Sohn W. Incidence of caries lesions among patients treated with comprehensive orthodontics. Am J Orthod Dentofacial Orthop. 2011;139(5):657-64. doi: 10.1016/j. ajodo.2009.06.037

11- Geiger AM. Mucogingival problems and the movement of mandibular incisors: a clinical review. Am J Orthod Dentofacial Orthop.1980;78(5):511-27. doi: 10.1016/0002-9416(80)90302-4 12- Weltman B, Vig KW, Fields HW, Shanker S, Kaizar EE. Root resorption associated with orthodontic tooth movement: a systematic review. Am J Orthod Dentofacial Orthop. 2010;137(4):462-76. doi: 10.1016/j.ajodo.2009.06.021

13- Grünheid T, Morbach BA, Zentner A. Pulpal cellular reactions to experimental tooth movement in rats. Oral Surg Oral Med Oral Pathol Oral Radiol Endod. 2007;(104):434-41. doi: 10.1016/j. tripleo.2007.03.022

14- Frost HM. The regional acceleratory phenomenon: a review. Henry Ford Hosp Med J.1983;31(1):3-9.

15- Yaffe A, Fine N, Binderman I. Regional accelerated phenomenon in the mandible following mucoperiosteal flap surgery. J Periodontol. 1994;65 (1):79-83. doi: 10.1902/jop.1994.65.1.79

16- Sebaoun JD, Kantarci A, Turner JW, Carvalho RS, Van Dyke TE, Ferguson DJ. Modeling of trabecular bone and lamina dura following selective alveolar decortication in rats. J Periodontol. 2008;79(9):167988. doi: 10.1902/jop.2008.080024

17- Baloul SS, Gerstenfeld LC, Morgan EF, Carvalho RS, Van Dyke TE, Kantarci A. Mechanism of action and morphologic changes in the alveolar bone in response to selective alveolar decortication-facilitated tooth movement. Am J Orthod Dentofacial Orthop. 2011;139(4 Suppl):S83-101. doi: 10.1016/j.ajodo.2010.09.026

18- Dibart S, Yee C, Surmenian J, Sebaoun JD, Baloul S, GoguetSurmenian $\mathrm{E}$, et al. Tissue response during Piezocision-assisted tooth movement: a histological study in rats. Eur J Orthod. 2014;36(4):45764. doi: 10.1093/ejo/cjt079

19- Patterson BM, Dalci O, Darendeliler MA, Papadopoulou AK. Corticotomies and orthodontic tooth movement: a systematic review. J Oral Maxillofac Surg. 2016;74(3):453-73. doi: 10.1016/j. joms.2015.10.011

20- Zuppardo ML, Ferreira CL, Moura NB, Longo M, Santamaria M Jr, Lopes $S \mathrm{~L}$, et al. Macroscopic and radiographic aspects of orthodontic movement associated with corticotomy: animal study. Oral Maxillofac Surg 2019;23(1):77-82. doi: 10.1007/s10006-019-00744-7 
21- Ferreira CL, Nunes CM, Bernardo DV, Pedroso JF, Longo M, Santamaria $\mathrm{M} \mathrm{Jr}$, et al. Effect of orthodontic force associated with cigarette smoke inhalation in healthy and diseased periodontium: a histometric and immunohistochemistry analysis in rats. J Periodontal Res. 2018;53(5):924-31. doi: 10.1111/jre.12584

22- Santamaria M Jr, Milagres D, Stuani AS, Stuani MB, Ruellas AC. Initial changes in pulpal microvasculature during orthodontic tooth movement: a stereological study. Eur J Orthod. 2006;28(3):217-20. doi: $10.1093 /$ ejo/cji117

23- Cho KW, Cho SW, OH CO, Ryu YK, Ohshima H, Jung HS. The effect of cortical activation on orthodontic tooth movement. Oral Dis. 2007;13(3):314-9. doi: 10.1111/j.1601-0825.2006.01286.x

24- Dutra EH, Ahmida A, Lima A, Schneider S, Nanda R, Yadav S. The effects of alveolar decortications on orthodontic tooth movement and bone remodelling in rats. Eur J Orthod. 2018;40(4):423-9. doi: 10.1093/ejo/cjx080

25- Nogueira AV, Molon RS, Nokhbehsaim M, Deschner J, Cirelli JA. Contribution of biomechanical forces to inflammation-induced bone resorption. J Clin Periodontol. 2017;44(1):31-41. doi: 10.1111/ jcpe. 12636

26- Santamaria-Jr M, Bagne L, Zaniboni E, Santamaria MP, Jardini MA Felonato $M$, et al. Diabetes mellitus and periodontitis: inflammatory response in orthodontic tooth movement. Orthod Craniofac Res. 2020;23(1):27-34. doi: 10.1111/ocr.12340

27- Librizzi Z, Kalajzic Z, Camacho D, Yadav S, Nanda R, Uribe F. Comparison of the effects of three surgical techniques on the rate of orthodontic tooth movement in a rat model. Angle Orthod. 2017;87(5):717-24. doi: 10.2319/123016-940.1
28- Peron AP, Johann AC, Papalexiou V, Tanaka OM, Guariza-Filho O, Ignácio $\mathrm{SA}$, et. al. Tissue responses resulting from tooth movement surgically assisted by corticotomy and corticision in rats. Angle Orthod. 2017;87(1):118-24. doi: 10.2319/102915-731.1

29- Tsai CY, Yang TK, Hsieh HY, Yang LY. Comparison of the effects of micro-osteoperforation and corticision on the rate of orthodontic tooth movement in rats. Angle Orthod. 2016;86(4):558-64. doi: 10.2319/052015-343.1

30- Hong RK, Yamane A, Kuwahara Y, Chiba M. The effect of orthodontic retention on the mechanical properties of the periodontal ligament in the rat maxillary first molar. J Dent Res. 1992;71(7):1350-4. doi: 10.1177/00220345920710070101

31- Verna C, Dalstra M, Melsen B. The rate and the type of orthodontic tooth movement is influenced by bone turnover in a rat model. Eur ] Orthod. 2000;22(4):343-52. doi: 10.1093/ejo/22.4.343

32- Ferreira CL, Rocha VC, Ursi WJ, Marco AC, Santamaria M Jr, Santamaria MP, et al. Periodontal response to orthodontic tooth movement in diabetes- induced rats with or without periodontal disease. J Periodontol. 2018;89(3):341-50. doi: 10.1002/JPER.17-0190 33- Boyce BF, Xing L. Biology of RANK, RANKL, and osteoprotegerin. Arthritis Res Ther. 2007; 9 Suppl 1:S1. doi: 10.1186/ar2165

34- Theoleyre S, Wittrant Y, Tat SK, Fortun Y, Redini F, Heymann D. The molecular triad OPG/RANK/RANKL: involvement in the orchestration of pathophysiological bone remodeling. Cytokine Growth Factor Rev. 2004;15(6):457-75. doi: 10.1016/j.cytogfr.2004.06.004 